PROCEEDINGS OF THE

AMERICAN MATHEMATICAL SOCIETY

Volume 139, Number 3, March 2011, Pages 1115-1120

S 0002-9939(2010)10600-7

Article electronically published on August 12, 2010

\title{
SYNDETIC SETS, PAVING AND THE FEICHTINGER CONJECTURE
}

\author{
VERN I. PAULSEN
}

(Communicated by Michael T. Lacey)

\begin{abstract}
We prove that if a Bessel sequence in a Hilbert space that is indexed by a countably infinite group in an invariant manner can be partitioned into finitely many Riesz basic sequences, then each of the sets in the partition can be chosen to be syndetic. We then apply this result to prove that if a Fourier frame for a measurable subset of a higher dimensional cube can be partitioned into Riesz basic sequences, then each subset can be chosen to be a syndetic subset of the corresponding higher dimensional integer lattice. Both of these results follow from a result about syndetic pavings of elements of the von Neumann algebra of a discrete group.
\end{abstract}

\section{INTRODUCTION}

Recently, W. Lawton [7 proved that given $A \subseteq[0,1]$ a set of positive Lebesgue measure, then the Fourier frame $\left\{e^{2 \pi i n t} \chi_{A}\right\}_{n \in \mathbb{Z}}$ for $L^{2}(A)$ can be partitioned into Riesz basic sequences if and only if there exists a syndetic subset of $S \subseteq \mathbb{Z}$ such that $\left\{e^{2 \pi i n t} \chi_{A}: n \in S\right\}$ is a Riesz basic sequence. In my dynamical systems approach to the Kadison-Singer problem [8], I proved that an operator in the von Neumann algebra of a countable abelian group that could be paved in the sense of Anderson could be paved by syndetic sets. Although this fact was derived in the proof of 8 , Theorem 16], it is not apparent from the statement of the theorem and has only been announced without proof at various meetings. If one combines this result about the syndetic paving of elements of the von Neumann algebra of a group with the method of proof of the equivalence of the Kadison-Singer conjecture and the Feichtinger conjecture [2], then one arrives, in this roundabout way, at a result that generalizes Lawton's result.

In this paper we make this argument direct. We begin with a direct proof of the result about the syndetic paving of elements of von Neumann algebras of groups (Theorem 2.3). This direct proof has the added advantage that we are able to eliminate the hypothesis that the group is abelian. We then show how this paving result directly yields statements about partitioning certain Bessel sequences into Riesz basic sequences. In particular, in addition to the paving theorem, we shall prove the following two theorems:

Received by the editors April 12, 2010.

2000 Mathematics Subject Classification. Primary 46L05; Secondary 46 B15.

This research was supported in part by NSF grant DMS-0600191 and by the American Institute of Mathematics.

(C)2010 American Mathematical Society Reverts to public domain 28 years from publication 
Theorem 1.1. Let $G$ be a countable group, let $\mathcal{H}$ be a Hilbert space and let $\left\{f_{g}\right\}_{g \in G}$ be a Bessel sequence in $\mathcal{H}$ with the property that for any $g, h, k \in G$, we have $\left\langle f_{g}, f_{h}\right\rangle=\left\langle f_{g k}, f_{h k}\right\rangle$. If $G$ can be partitioned into finitely many subsets $\left\{A_{l}: 1 \leq\right.$ $l \leq L\}$ such that for $1 \leq l \leq L,\left\{f_{g}: g \in A_{l}\right\}$ is a Riesz basic sequence, then $G$ can be partitioned into $K$ syndetic subsets $\left\{S_{k}: 1 \leq k \leq K\right\}$ with $K \leq L$ such that for $1 \leq k \leq K,\left\{f_{g}: g \in S_{k}\right\}$ is a Riesz basic sequence.

Note that the condition on the inner products in the above theorem is equivalent to requiring that for any $g_{1}, g_{2}, h_{1}, h_{2} \in G$ satisfying $g_{1} g_{2}^{-1}=h_{1} h_{2}^{-1}$ we have $\left\langle f_{g_{1}}, f_{g_{2}}\right\rangle=\left\langle f_{h_{1}}, f_{h_{2}}\right\rangle$.

To state the second theorem it will be convenient to introduce some notation. Given a natural number $N$, we let $[0,1]^{N}$ denote the product of $N$ copies of the unit interval and we let $\mathbb{Z}^{N}$ denote the group that is the direct sum of $N$ copies of the group of integers. If $t=\left(t_{1}, \ldots, t_{N}\right) \in[0,1]^{N}$ and $n=\left(n_{1}, \ldots, n_{N}\right) \in \mathbb{Z}^{N}$, then we set $n \cdot t=n_{1} t_{1}+\cdots+n_{N} t_{N}$ and set $e_{n}(t)=e^{2 \pi i n \cdot t}$. We have that $\left\{e_{n}(t): n \in \mathbb{Z}^{N}\right\}$ is an orthonormal basis for the Hilbert space $L^{2}\left([0,1]^{N}\right)$ of square-integrable functions with respect to Lebesgue product measure. If $A \subseteq[0,1]^{N}$ is a set of positive Lebesgue measure and $L^{2}(A)$ denotes the corresponding Hilbert space of squareintegrable functions on $A$, then $f_{n}(t)=e_{n}(t) \chi_{A}, n \in \mathbb{Z}^{N}$ is a Parseval frame for $L^{2}(A)$ called the Fourier frame for $L^{2}(A)$.

Theorem 1.2. Let $N$ be a natural number and let $A \subseteq[0,1]^{N}$ be a set of positive Lebesgue measure. If the Fourier frame $\left\{f_{n}\right\}_{n \in \mathbb{Z}^{N}}$ for $L^{2}(A)$ can be partitioned into finitely many subsets $\left\{A_{l}: 1 \leq l \leq L\right\}$ such that for $1 \leq l \leq L,\left\{f_{n}: n \in A_{l}\right\}$ is a Riesz basic sequence, then $\mathbb{Z}^{N}$ can be partitioned into $K$ syndetic subsets $\left\{S_{k}\right.$ : $1 \leq k \leq K\}$ with $K \leq L$ such that for $1 \leq k \leq K,\left\{f_{n}: n \in S_{k}\right\}$ is a Riesz basic sequence.

\section{Proofs of the main Results}

We begin by recalling some results and notation from 8 . Let $G$ be a countably infinite group, let $\ell^{2}(G)$ denote the Hilbert space of square-summable functions on $G$, let $\ell^{\infty}(G)$ denote the algebra of bounded functions on $G$, let $\beta G$ denote the Stone-Cech compactification of $G$ and let $C(\beta G)$ denote the algebra of continuous functions on $\beta G$. Every element of $\ell^{\infty}(G)$ extends uniquely to a continuous function on $\beta G$, and conversely, and hence, we identify $\ell^{\infty}(G)=C(\beta G)$. We let $e_{g} \in \ell^{2}(G)$ denote the function that is 1 at $g$ and 0 elsewhere, so that $\left\{e_{g}: g \in G\right\}$ is an orthonormal basis for $\ell^{2}(G)$. We let $B\left(\ell^{2}(G)\right)$ denote the space of bounded operators on $\ell^{2}(G)$ and given a bounded operator $X$ we identify $X$ with a $G \times G$ matrix by setting $X=\left(x_{g, h}\right)$, where $x_{g, h}=\left\langle X e_{h}, e_{g}\right\rangle$. If a bounded operator is diagonal with respect to this basis, then its diagonal entries define an element of $\ell^{\infty}(G)$, so we also identify $\ell^{\infty}(G) \subseteq B\left(\ell^{2}(G)\right)$ as the abelian subalgebra of diagonal operators. We let $E: B\left(\ell^{2}(G)\right) \rightarrow \ell^{\infty}(G)$ denote the completely positive projection defined by setting $E(X)$ equal to the diagonal part of the matrix $X$.

We let $\lambda: G \rightarrow B\left(\ell^{2}(G)\right)$ denote the left regular representation of $G$ given by $\lambda(g) e_{h}=e_{g h}$. The von Neumann algebra of the group is the double commutant of the left regular representation, $V N(G)=\lambda(G)^{\prime \prime}$.

Given $X=\left(x_{g, h}\right) \in B\left(\ell^{2}(G)\right)$, we set $D_{g}=E\left(X \lambda\left(g^{-1}\right)\right)$; that is, $D_{g}$ is the diagonal matrix $\left(y_{h, h}\right)$, where $y_{h, h}=x_{h, g^{-1} h}$. The operators $D_{g}$ can be thought of as the terms in a formal series for $X$, in the sense that $\sum_{g \in G} D_{g} \lambda(g)$ converges 
entrywise to the matrix for $X$. We shall write $X \sim \sum_{g \in G} D_{g} \lambda(g)$. It can be easily seen that $X \in V N(G)$ if and only if $X \sim \sum_{g \in G} c_{g} \lambda(g)$ for some constants $c_{g}$. That is, $X \in V N(G)$ if and only if $E\left(X \lambda\left(g^{-1}\right)\right)$ is a scalar multiple of the identity operator for every $g \in G$.

We also need to recall the semigroup structure of $\beta G$. For a good reference, see the text of Hindman-Strauss [6], but we also recall the salient points below. The action of $G$ on $G$ given by left translation extends to give an action of $G$ on $\beta G$. In particular, if $g \in G, \omega \in \beta G$, and $\left\{g_{\lambda}\right\}$ is a net in $G$ converging to $\omega$, then the net $\left\{g g_{\lambda}\right\}$ converges to the element $g \cdot \omega$. Moreover, this left action extends to define an associative product making $\beta G$ into a right continuous, compact semigroup. If $\alpha, \omega \in \beta G$, and $\left\{g_{\lambda}\right\},\left\{h_{\mu}\right\}$ are nets in $G$ with $\alpha=\lim _{\mu} h_{\mu}, \omega=\lim _{\lambda} g_{\lambda}$, then $\alpha \cdot \omega=\lim _{\mu}\left[\lim _{\lambda} h_{\mu} g_{\lambda}\right]$.

Fixing an element $\omega \in \beta G$, we defined in 8 a *-homomorphism, $\pi_{\omega}: C(\beta G) \rightarrow$ $C(\beta G)$, by setting $\pi_{\omega}(f)(g)=f(g \cdot \omega)$. Via our identifications, we also write $\pi_{\omega}(D)$, where $D \in \ell^{\infty}(G) \subseteq B\left(\ell^{2}(G)\right)$ is the diagonal operator that is identified with the function $f$. In 8 , we proved that there is a well-defined, unital completely positive map $\psi_{\omega}: B\left(\ell^{2}(G)\right) \rightarrow B\left(\ell^{2}(G)\right)$ defined as follows:

$$
\text { if } X \sim \sum_{g \in G} D_{g} \lambda(g), \text { then } \psi_{\omega}(X) \sim \sum_{g \in G} \pi_{\omega}\left(D_{g}\right) \lambda(g) .
$$

Given a subset $A \subseteq G$, we let $P_{A}$ denote the diagonal operator $\left(y_{h, h}\right)$ with $y_{h, h}=1$ when $h \in A$ and 0 , otherwise. That is, $P_{A}$ is the diagonal operator that is identified with the characteristic function $\chi_{A}$ of the set $A$.

In [6. Theorem 2.8] it is shown that the compact right topological semigroup $\beta G$ contains a non-empty minimal (two-sided) ideal, denoted $K(\beta G)$. Finally, recall that a subset $S \subseteq G$ is called syndetic if there exists a finite set $F \subseteq G$, such that $G=\bigcup_{g \in F} g^{-1} S$, i.e., $G=F^{-1} \cdot S$.

Lemma 2.1. Let $G$ be a countably, infinite discrete group, let $p \in K(\beta G)$, and let $A \subseteq G$. If $p \in K(\beta G)$, then $\pi_{p}\left(P_{A}\right)=P_{R}$, with $R$ either empty or syndetic.

Proof. Since $\pi_{p}$ is a $*$-homomorphism, $\pi_{p}\left(P_{A}\right)$ is a projection in $\ell^{\infty}(G)$ and hence, $\pi_{p}\left(P_{A}\right)=P_{R}$ for some subset $R \subseteq G$. If $R$ is non-empty, then there exists $g_{0} \in R$ and hence $1=\pi_{p}\left(P_{A}\right)\left(g_{0}\right)=f\left(g_{0} \cdot p\right)$, where $f$ denotes the continuous map of $P_{A}$ to $\beta G$. That is, $f$ is the characteristic function of the closure of $A, A^{-}$in $\beta G$. Thus, $g_{0} \cdot p \in A^{-}$, or $p \in\left(g_{0}^{-1} A\right)^{-}$. Thus, we have that $R=\left\{g \in G: p \in\left(g^{-1} A\right)^{-}\right\}$.

Using the identification of points in $\beta G$ with their corresponding ultrafilters, we see that this means that the set $g_{0}^{-1} A$ belongs to the ultrafilter $p$, i.e., $g_{0}^{-1} A \in p$. In this notation, $R=\left\{g \in G: g^{-1} A \in p\right\}$.

Since $p \in K(\beta G)$ and $g_{0}^{-1} A \in p$, by [6, Theorem 4.39], $S=\left\{x \in G: x^{-1} g_{0}^{-1} A \in\right.$ $p\}$ is syndetic. Hence, $R=g_{0} S$ is also syndetic.

We shall also need a consequence of a result of Choi's from the theory of multiplicative domains [3, Theorem 3.1].

Lemma 2.2 (Choi). Let $\mathcal{A}$ and $\mathcal{B}$ be unital $C^{*}$-algebras and let $\phi: \mathcal{A} \rightarrow \mathcal{B}$ be a unital, completely positive map. If $1_{\mathcal{A}} \in \mathcal{C} \subseteq \mathcal{A}$ is a $C^{*}$-subalgebra such that the restriction of $\phi$ to $\mathcal{C}$ is a ${ }^{*}$-homomorphism $\pi$, then for any $c_{1}, c_{2} \in \mathcal{C}$ and any $a \in \mathcal{A}$, we have that $\phi\left(c_{1} a c_{2}\right)=\pi\left(c_{1}\right) \phi(a) \pi\left(c_{2}\right)$. 
Proof. The result follows by applying [3, Theorem 3.1] to both $c$ and $c^{*}$ for $c \in$ $\mathcal{C}$.

We can now prove a result about paving elements of the von Neumann algebra of a group. For the case that $G$ is abelian, this result was announced at the 2008 Cincinnati GPOTS (Great Plains Operator Theory Symposium).

Theorem 2.3. Let $G$ be a countably infinite discrete group, let $X \in V N(G)$ with $E(X)=0$, and let $\epsilon<1$. If $G$ can be partitioned into finitely many subsets $\left\{A_{l}\right.$ : $1 \leq l \leq L\}$ such that for $1 \leq l \leq L,\left\|P_{A_{l}} X P_{A_{l}}\right\| \leq \epsilon\|X\|$, then $G$ can be partitioned into $K$ syndetic subsets $\left\{S_{k}: 1 \leq k \leq K\right\}$ with $K \leq L$ such that for $1 \leq k \leq K$, $\left\|P_{S_{k}} X P_{S_{k}}\right\| \leq \epsilon\|X\|$.

Proof. Fix any $p \in K(\beta G)$. Then we have a unital, completely positive map, $\psi_{p}: B\left(\ell^{2}(G)\right) \rightarrow B\left(\ell^{2}(G)\right)$. Since such maps are contractive, we have that $\left\|\psi_{p}\left(P_{A_{l}} X P_{A_{l}}\right)\right\| \leq \epsilon\|X\|$. Note that if $D \in \ell^{\infty}(G)$ is a diagonal operator, then $\psi_{p}(D)=\pi_{p}(D)$, and so by Choi's Lemma, $\psi_{p}\left(P_{A_{l}} X P_{A_{l}}\right)=\pi_{p}\left(P_{A_{l}}\right) \psi_{p}(X) \pi_{p}\left(P_{A_{l}}\right)$.

By the first lemma, $\pi_{p}\left(P_{A_{l}}\right)=P_{S_{l}}$, where $S_{l}$ is either syndetic or empty. Also, since $\pi_{p}$ is a unital ${ }^{*}$-homomorphism, $I=\pi_{p}\left(P_{A_{1}}+\cdots+P_{A_{L}}\right)=P_{S_{1}}+\cdots+P_{S_{L}}$, which implies that $G$ is the disjoint union of the non-empty syndetic sets.

Finally, note that since $X \in V N(G)$, there exist scalars such that $X \sim$ $\sum_{g \in G} c_{g} I \lambda(g)$, and hence, $\psi_{p}(X) \sim \sum_{g \in G} \pi_{p}\left(c_{g} I\right) \lambda(g) \sim X$, that is, $\psi_{p}(X)=X$. Thus, we have that $P_{S_{l}} X P_{S_{l}}=\psi_{p}\left(P_{A_{l}} X P_{A_{l}}\right)$, and the result follows.

Remark 2.4. In the case that $G=\mathbb{Z}^{N}$ the Fourier transform defines an isomorphism between $\ell^{2}\left(\mathbb{Z}^{N}\right)$ and $L^{2}\left([0,1]^{N}\right)$. That is, choosing the orthonormal basis $\left\{e^{2 \pi i n \cdot t}\right.$ : $\left.n \in \mathbb{Z}^{N}\right\}$, defines the Hilbert space isomorphism. This allows the identification, $B\left(\ell^{2}\left(\mathbb{Z}^{N}\right)\right)=B\left(L^{2}\left([0,1]^{N}\right)\right)$ and with respect to this identification, $V N\left(\mathbb{Z}^{N}\right)=$ $\left\{M_{f}: f \in L^{\infty}\left([0,1]^{N}\right)\right\}$, where $M_{f}$ denotes the operator of multiplication by the function $f$. The operator $M_{f}$ is often called the Laurent operator with symbol $f$, especially in the case $N=1$. Thus, the above theorem, specialized to the case of $\mathbb{Z}^{N}$, shows that if a Laurent operator can be paved, then it can be paved by syndetic sets in the sense of the above theorem.

Thus to find a Laurent operator that is not pavable and hence show that the Kadison-Singer problem has a negative answer, it would be enough to find a Laurent operator that cannot be syndetically paved in the above sense.

We now turn our attention to the case of Bessel sequences in Hilbert spaces. We need one observation.

Lemma 2.5. Let $\left\{f_{i}\right\}_{i \in I}$ be a Bessel sequence in a Hilbert space and let $A \subseteq I$. Then $\left\{f_{i}\right\}_{i \in A}$ is a Riesz basic sequence if and only if there exists $\delta>0$ such that the operator inequality $\delta P_{A} \leq P_{A}\left(\left\langle f_{j}, f_{i}\right\rangle\right) P_{A}$ holds on $B\left(\ell^{2}(I)\right)$.

We can now prove the results announced in the introduction.

Proof of Theorem 1.1. Let $X=\left(x_{g, h}\right)=\left(\left\langle f_{h}, f_{g}\right\rangle\right)$ and note that since $\left\{f_{g}\right\}_{g \in G}$ is a Bessel sequence, $X$ is the matrix of a bounded, positive operator on $B\left(\ell^{2}(G)\right)$. The condition $\left\langle f_{h}, f_{g}\right\rangle=\left\langle f_{h k}, f_{g k}\right\rangle$ guarantees that $X \in V N(G)$. To see this, note that $X \in V N(G)$ if and only if for every $g \in G, E(X \lambda(g))$ is a scalar multiple of the identity. We have that

$$
\left\langle X \lambda(g) e_{h}, e_{h}\right\rangle=x_{h, g h}=\left\langle f_{g h}, f_{h}\right\rangle=\left\langle f_{g}, f_{e}\right\rangle,
$$


where $e$ denotes the identity element of $G$. Thus, $E(X \lambda(g))=c_{g} I$, where $c_{g}=$ $\left\langle f_{g}, f_{e}\right\rangle$ and, consequently, $X \sim \sum_{g \in G} c_{g} \lambda(g)$.

Now since $\left\{f_{g}: g \in A_{l}\right\}$ is a Bessel sequence for $1 \leq l \leq L$, there exists $\delta>0$, such that $\delta P_{A_{l}} \leq P_{A_{l}} X P_{A_{l}}$, for all $l$. Fix any $p \in K(\beta G)$ and apply $\psi_{p}$ to this operator inequality, to obtain $\delta P_{S_{l}}=\delta \psi_{p}\left(P_{A_{l}}\right) \leq \psi_{p}\left(P_{A_{l}} X P_{A_{l}}\right)=P_{S_{l}} X P_{S_{l}}$, where each $S_{l}$ is either empty or syndetic.

The inequality $\delta P_{S_{l}} \leq P_{S_{l}} X P_{S_{l}}$ guarantees that $\left\{f_{g}: g \in S_{l}\right\}$ is a Riesz basic sequence, and so the proof is complete.

Proof of Theorem 1.2, We shall apply Theorem 1.1 with $G=\mathbb{Z}^{N}$. Note that for any $n, m \in \mathbb{Z}^{N}$, we have that

$$
\left\langle f_{n}, f_{m}\right\rangle=\int_{A} e_{n-m}(t) d t=\left\langle f_{n-m}, f_{0}\right\rangle,
$$

where $d t$ denotes the $N$-dimensional Lebesgue measure. From this identity it readily follows that the Bessel sequence $\left\{f_{n}: n \in \mathbb{Z}^{N}\right\}$ satisfies the necessary group invariance condition. So the hypotheses of Theorem 1.1 are met and the result follows.

Note that the essence of the proof of Theorem 1.2 rests on the fact that the Grammian of the Fourier frame for the set $A$ is the matrix of the Laurent operator whose symbol is the characteristic function of $A$.

Remark 2.6. Let $S \subseteq \mathbb{Z}^{N}$ be a syndetic set and let $F \subseteq \mathbb{Z}^{N}$ be a finite set such that $F+S=\mathbb{Z}^{N}$. For $n=\left(n_{1}, \ldots, n_{N}\right) \in \mathbb{Z}^{N}$, set $\|n\|=\max \left\{\left|n_{1}\right|, \ldots,\left|n_{N}\right|\right\}$, i.e., the supremum norm. Let $M=\max \{\|m\|: m \in F\}+1$. Then given any $n \in S$, there must exist $m \in S, m \neq n$, with $\|n-m\| \leq M$. That is, every $N$-cube with side of length $M$ must contain at least one element of $S$. It is well known and easily seen that this last property is a characterization of syndetic sets in $\mathbb{Z}^{N}$. This property implies that $S$ will have positive "density" for most reasonable definitions of density. In particular, for $N=1$ a set is syndetic if and only if it has a uniformly bounded gap between adjacent elements and, consequently, has positive lower Beurling density. Combining these observations with our earlier remark, we see that if a Laurent operator can be paved, then it can be paved with syndetic sets and consequently with sets of positive lower Beurling density. Our results should be compared with the classic result of Bourgain and Tzafriri [1, Theorem 2.2], which says that for the Fourier frames and $N=1$, there always exists a subset of $\mathbb{Z}$ of positive density that is a Riesz basic sequence. But [1] uses a much larger definition of density, and sets of integers can have positive density in their sense and fail to have bounded gap size, i.e., fail to be syndetic.

Remark 2.7. In the case $G=\mathbb{Z}$, Halpern, Kaftal and Weiss [5] studied uniform paving of Laurent operators. That is, they asked when $M_{f} \in B\left(\ell^{2}(\mathbb{Z})\right)$ with $E\left(M_{f}\right)=0$ had the property that for each $\epsilon>0$, there existed an $M>0$, such that if one let $A=\{M n: n \in \mathbb{Z}\}$, then $\left\|P_{A} M_{f} P_{A}\right\|<\epsilon\left\|M_{f}\right\|$. They characterized the symbols for which this could occur as the functions for which certain "uniform averages" of the function converged uniformly to a constant function.

In contrast our result shows that if the paving of a Laurent operator can be done at all, then the set $A$ can be chosen to be syndetic and hence, while not uniform, it will have a "bounded gap"; i.e., it must select at least one element from each interval of length $M$, for some $M$. 
This last remark lends credence to the belief that the symbols for which $M_{f}$ is pavable are perhaps a proper subspace of $L^{\infty}([0,1])$. Perhaps, the symbols that can be paved have a characterization in terms of uniform convergence of non-uniform averages.

The following result gives another characterization of sequences of vectors that satisfy the group invariance conditions of Theorem 1.1 .

Proposition 2.8. Let $G$ be a group, let $\mathcal{H}$ be a Hilbert space and let $\left\{f_{g}\right\}_{g \in G}$ be a Bessel family, whose closed linear span is $\mathcal{H}$. Then $\left\langle f_{g}, f_{h}\right\rangle=\left\langle f_{g k}, f_{h k}\right\rangle$ for any $g, h, k \in G$ if and only if there is a unitary representation $\pi: G \rightarrow B(\mathcal{H})$ satisfying $\pi(g) f_{h}=f_{h g^{-1}}$ for all $g, h \in G$.

Proof. Assume that such a unitary representation exists and let $g, h, k \in G$. Then

$$
\left\langle f_{g}, f_{h}\right\rangle=\left\langle\pi\left(k^{-1}\right) f_{g}, \pi\left(k^{-1}\right) f_{h}\right\rangle=\left\langle f_{g k}, f_{h k}\right\rangle .
$$

Conversely, assume that the Bessel family satisfies the invariance condition and fix $g \in G$. Recall that the analysis operator of the set is the bounded linear operator $V: \mathcal{H} \rightarrow \ell^{2}(G)$ given by $(V x)(s)=\left\langle x, f_{s}\right\rangle$ for $x \in \mathcal{H}$ and $s \in G$. The adjoint of this operator satisfies $V^{*} e_{s}=f_{s}$, where $e_{s} \in \ell^{2}(G)$ is the characteristic function of the singleton $s$. If we also define $V_{g}: \mathcal{H} \rightarrow \ell^{2}(G)$, by $\left(V_{g} x\right)(s)=\left\langle x, f_{s g^{-1}}\right\rangle$, then $V_{g}^{*} e_{s}=f_{s g^{-1}}$. Hence, for any $s, t \in G,\left\langle V_{g} V_{g}^{*} e_{t}, e_{s}\right\rangle=\left\langle f_{t g^{-1}}, f_{s g^{-1}}\right\rangle=\left\langle f_{t}, f_{s}\right\rangle=$ $\left\langle V V^{*} e_{t}, e_{s}\right\rangle$. Thus, $V_{g} V_{g}^{*}=V V^{*}$ and so by Douglas' factorization theorem [4, there is a unitary operator $R_{g}: \mathcal{H} \rightarrow \mathcal{H}$ such that $R_{g} V^{*}=V_{g}^{*}$. Thus, we have that $R_{g} f_{s}=f_{s g^{-1}}$, for every $s \in G$, and from this relation it follows that the map $\pi(g)=R_{g}$ defines a unitary representation of $G$ on $\mathcal{H}$.

\section{REFERENCES}

[1] J. Bourgain and L. Tzafriri, Invertibility of "large" submatrices with applications to the geometry of Banach spaces and harmonic analysis, Israel J. of Math. 57, no. 2 (1987), 137-224. MR890420 (89a:46035)

[2] P. Casazza and J. Tremain, The Kadison-Singer problem in mathematics and engineering, Proc. Nat. Acad. of Sciences 103, no. 7 (2006), 2032-2039. MR2204073 (2006j:46074)

[3] M.D. Choi, A Schwarz inequality for positive linear maps on $C^{*}$-algebras, Illinois J. Math. 18 (1974), 565-574. MR0355615 (50:8089)

[4] R.G. Douglas, On majorization, factorization and range inclusion of operators in Hilbert space, Proc. Amer. Math. Soc. 17 (1966), 413-416. MR0203464 (34:3315)

[5] H. Halpern, V. Kaftal and G. Weiss, Matrix pavings and Laurent operators, J. Operator Theory 16 (1986), no. 2, 355-374. MR860353 (87j:46106)

[6] N. Hindman and D. Strauss, Algebra in the Stone-Cech Compactification, De Gruyter Expositions in Mathematics, 27, de Gruyter Publishing, New York, 1998. MR.1642231 (99j:54001)

[7] W. Lawton, Minimal sequences and the Kadison-Singer problem, Bulletin of the Malaysian Math. Soc. (2) 33(2) (2010), 169-176.

[8] V.I. Paulsen, A dynamical systems approach to the Kadison-Singer problem, J. Funct. Anal. 255 (2008), 120-132. MR2417811 (2009b:46126)

Department of Mathematics, University of Houston, Houston, Texas 77204-3476

E-mail address: vern@math.uh.edu 